\title{
Assessment of Power Plant Based on Unsafe Behavior of Workers through Backpropagation Neural Network Model
}

\author{
Juan Shi $\mathbb{D}$ and Dingyi Chang \\ School of Management, Tianjin University of Technology, Tianjin 300384, China \\ Correspondence should be addressed to Juan Shi; 422080847@qq.com and Dingyi Chang; changdingyi@stud.tjut.edu.cn
}

Received 10 November 2021; Revised 17 December 2021; Accepted 23 December 2021; Published 12 January 2022

Academic Editor: Hasan Ali Khattak

Copyright (C) 2022 Juan Shi and Dingyi Chang. This is an open access article distributed under the Creative Commons Attribution License, which permits unrestricted use, distribution, and reproduction in any medium, provided the original work is properly cited.

\begin{abstract}
Safety is an essential topic for electric power plants. In recent years, accidents caused by unsafe behaviors of electric power plant employees are frequent. To promote the sustainable development and safety of electric power plants, studies on the assessment of unsafe behavior are becoming increasingly important and urgent. In this study, accident statistical analysis, literature review, and expert survey are adopted to select more comprehensive and accurate assessment indicators of unsafe behavior of the workers in electric power plants. Data about indicator and unsafe behavior were obtained through a questionnaire survey, and 27 indicators were used as inputs, and the unsafe behavior was taken as the output of a backpropagation (BP) neural network based unsafe behavior assessment model. An assessment indicator system about power plant workers' unsafe behavior composed of 4 first-level indicators and 27 second-level indicators was established and the weights of the assessment indicators were determined. A threelayer feedforward BP neural network assessment model of "27-13-1" layers was found to be a suitable model. The proposed model can demonstrate the nonlinear complex relationship between the assessment indicator and the unsafe behavior of power plant workers. The model can be helpful to evaluate, predict, and monitor the safety performance of electric power plants.
\end{abstract}

\section{Introduction}

Electrical accidents of the workers in the electric power industry can be lethal and fatal. Although electrical professional's electrical safety in China is treated at a high level, accidents do happen. Moreover, electrical accidents are not as rare as statistics imply as many minor accidents remain unreported [1]. Underreporting causes a lack of information about existing electrical safety problems and prevents the corresponding preventive measures. As long as accidents happen, this implies that current measures to increase electrical safety are not effective enough. With the advances in mechanization, automation, and intelligence in power plants, accidents caused by equipment malfunction are reducing, whereas an increasing number of accidents result from human errors and unsafe behavior [2]. The unsafe behaviors include wrongful operation, ignorance of warnings, nonuse of safety devices, use of unsafe equipment, restricted area entering, and failure to meet dress code standards. For example, in May 2021, an operator of Gansu Electric Power Company failed to buckle his seat belt and died from a fall. In June 2020, a construction personnel of Guangxi Electric Power Company died from electric shock with no prior power failure inspection [3]. The analysis shows that the direct cause of the majority of the accidents is the employees' wrongful operation and unsafe behavior, and these accidents have a negative impact on the enterprise and even on the society. Therefore, it is of great significance to prevent and control the unsafe behavior of power plant workers to reduce the occurrence of human accidents [4].

Prevention and control of unsafe behaviors require comprehensive and accurate assessment. However, due to the uncertainty and repeatability of unsafe behaviors, the assessment becomes complicated. Up to now numerous scholars have carried out related research on the assessment of unsafe behavior. Malakoutikhah et al. [5] used grounded theory and interviewed 40 workers with a semistructured interview method. The results showed that organizational 
factors played important roles in the assessment of unsafe behaviors. Mokarami et al. [6] studied the relationship between bus drivers' safety culture, unsafe behaviors, and traffic accidents using constructing a structural equation model. It was found that there is a negative relationship between safety culture level and accidents rate, and drivers' unsafe behaviors frequency and unsafe behavior play a significant intermediate role between safety culture and accidents. The author Shin et al. [7] built a mental process model of construction workers based on the system dynamics (SD) method and then examined the feedback mechanism of the safety attitude and unsafe behavior. An assessment factor system of unsafe behavior and an analytic hierarchy process model were proposed by Li et al. [8]. For quantification of behavioral safety data of workers, the fuzzy assessment method was employed. Tong et al. [9] proposed a risk assessment model and used the Monte Carlo method to calculate the risk value of employees' unsafe behaviors. Zhang et al. [10] employed the Planned Behavior theory and explored the effects of nuclear power plant workers' attitudes, perceptions, and subjective norms on unsafe behaviors. Yu et al. [11] used the Fishbone Diagram and established an assessment model for the influencing factors of coal mine workers' unsafe behaviors from five aspects: individual, physical environment, safety leadership, safety management, and group organization. In addition, there are many studies on the assessment of unsafe behavior, which guides subsequent studies. However, the existing research lacks robustness, relevance, and differentiation of assessment indicators for unsafe behaviors. Moreover, the method of acquiring assessment indicators is relatively ineffective and the weights are mostly calculated by cause-to-effect methods such as analytic hierarchy process, grey correlation degree, expert survey method, and entropy weight method which are affected by randomness and human factors during the assessment process.

As a cause-and-effect method, machine learning algorithms can mimic human thinking models and effectively deal with nonlinear prediction problems. In recent years, machine learning algorithms such as backpropagation (BP) neural network have been widely used in the field of assessment. Using BP neural network, Li and Wang [12] evaluated the safety of subway construction projects under construction from three aspects including human factors, management factors, and risk factors and then predicted the possibility of accidents. Tseng and Luo [13] developed an employee quality assessment model based on a BP neural network to rate the performance through the collection and processing of employee performance data. Han et al. [14] employed a genetic algorithm to optimize BP neural network modeling and built a hybrid genetic BP model, to efficiently evaluate and select the product design. Likewise, Feng et al. [15] identified the possible risk factors in the supply chain and constructed the risk assessment indicator system based on an optimized BP neural network. Zhang et al. [16] proposed an assessment indicator system for the information communicability of think tank WeChat public platform based on BP neural network from four dimensions, including the think tank power, information content features, public platform's service capability, and subscribers' performance. Although BP neural network has made great achievements in many fields, the application of the BP algorithm in the assessment of power plant workers' unsafe behavior is still in infancy [17]. This study adopted accident statistical analysis, literature review, and expert survey methods to select more comprehensive and accurate assessment indicators of unsafe behavior. Based on the data of unsafe behavior of electric power plant workers in Tianjin, Yinchuan, Zoucheng, and Yangquan cities of China, a BP neural network assessment model is developed. The weight of the assessment indicator is calculated and analyzed, to provide advice for the prevention and control of power plant workers' unsafe behaviors and firmly help uphold the enterprise "Safety First" culture.

The rest of the manuscript is arranged as follows: Section 2 provides detail about the data collection and analysis process. In Section 3, the results are illustrated. Section 4 is about discussion and the conclusion and limitations are presented in Section 5.

\section{Materials and Methods}

2.1. Research Process. Safe, reliable, and inexpensive electrical energy is essential to the world economy and our way of life. Electricity powers our homes, offices, and industries, provides entertainment, medical services, and communication, and powers various technologies. This study aims to evaluate, prevent, and control the unsafe behaviors of power plant workers based on practical problems. The framework of the proposed research is shown in Figure 1. There are six steps within the research: problem discovery, selection of assessment indicators, collection of data, determination of BP neural network structure, model simulation, and discussion. In this way, the weights of unsafe behavior assessment indicators can be determined, and prevention and control measures can be formulated and implemented. These steps are illustrated in the subsequent sections.

\subsection{Selection of Assessment Indicators}

(i) Accident Statistical Analysis. According to the public information of the Chinese National Energy Administration, the Chinese Ministry of Emergency Management and Safety Management Network, the data of production safety accidents in the power industry from 2015 to 2019 were collected and sorted out and 145 personal injury accidents were selected, including 39 cases of falling from a height, 26 cases of electric shock, 22 cases of object hit, 19 cases of mechanical injury, 14 cases of lifting injury, 13 cases of collapse, and 12 cases of others. In the 145 accidents, the unsafe state of objects accounted for $37 \%$ of the total accidents, and the unsafe behavior of people accounted for $63 \%$. The accidents caused by unsafe behaviors are analyzed statistically, the influencing factors of unsafe behaviors are extracted, and then the assessment indicators are determined. 


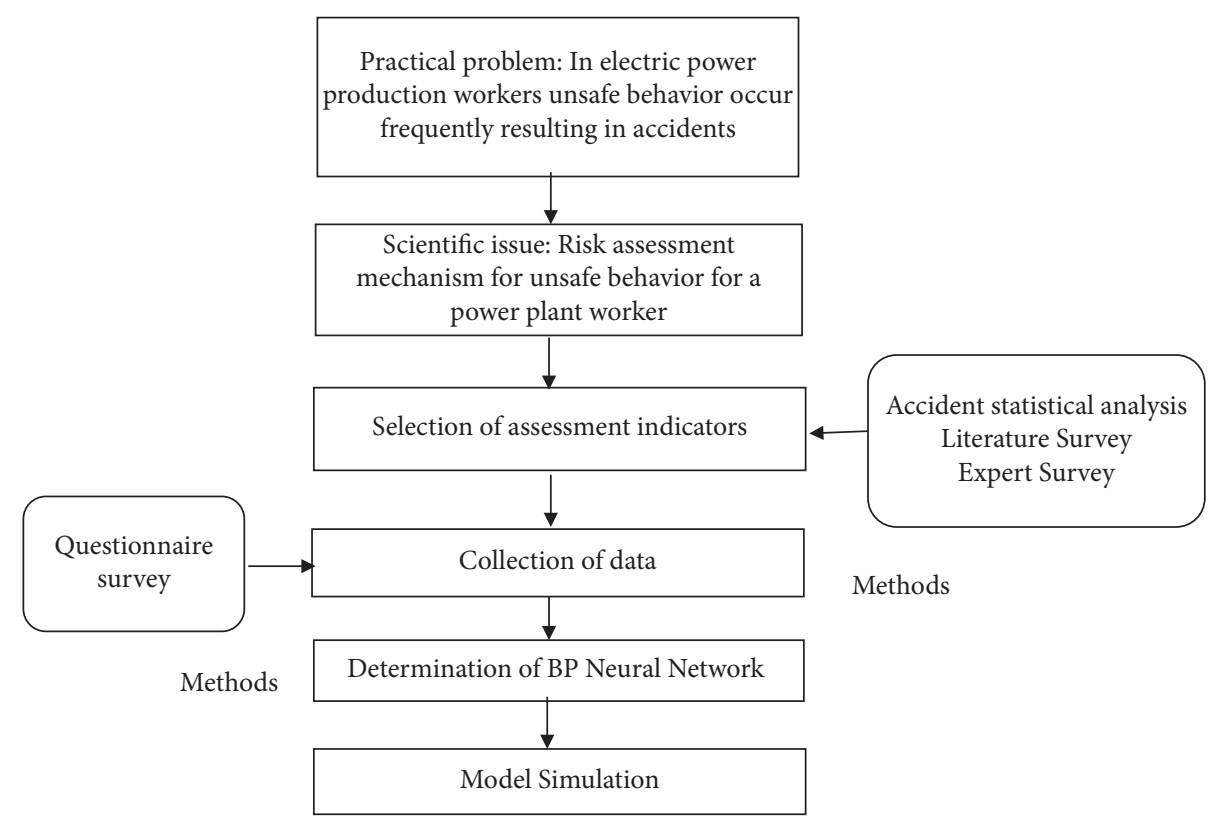

FIgURE 1: Model framework.

(ii) Literature Review. Using "Web of Science," "Elsevier ScienceDirect," and "CNKI" as literature retrieval databases, the keywords were selected as "unsafe behavior" or "violation behavior" or "risk behavior" or "safe behavior" or "crisis behavior." The topic was selected as "power plant workers" or "power plant operators" or "electric power employees," and the retrieval time was limited to 2019-2021. More than 1000 journal papers were selected. By reading the title, abstract, and keywords of the papers, the searching criteria were further simplified, and we finally got 121 journal papers. The influencing factors of unsafe behaviors are clarified and summarized.

According to the results obtained by the above methods, the assessment indicator's initial set of unsafe behavior was obtained by selecting and categorizing, as shown in Table 1.

(iii) Expert Survey Method. It is a relatively mature method for indicator determination, which can promote anonymous communication among experts, integrate opinions, and reach a relatively consistent view [18]. According to the knowledge and experience of experts, this method carries out multiple rounds of questionnaire surveys and collates the results to determine the final answer, which has a strong scientific nature. According to Table 1, this study used an expert survey method to score the indicators and consulted experts to further supplement and simplify the indicators.

The assessment object of this study is the unsafe behavior of power plant workers. To this end, the author invited 8 experts from Huadian Electric Power Research Institute, Tianjin University, Nankai University, and other enterprises and universities. All the experts have research achievements in the field of safety management and have a certain understanding of the unsafe behavior of power plant workers. Three rounds of questionnaires were issued. The first round of questionnaires was all collected (12 copies), and the experts optimized the existing indicators. Less important indicators, such as monotonousness, readiness, inspection, biological hazard, work time, and social norm, were dropped. Emotional management ability, safety awareness, regulation, safety supervision, and other indicators were highly recognized. In addition, an expert divided the existing indicators into five first-level indicators: individual, organization, equipment, management, and environment. An expert suggested combining indicators such as high temperature, ventilation, visibility, and odor into a single indicator called physical environmental physical fitness indicator. An expert believed that the three indicators of leadership and management ability, management commitment, and management level have similar meanings and can be collectively changed to management ability. The results of the first round of questionnaires were collected and the second round of the survey was conducted.

The second round of questionnaires was all recovered, and experts gave suggestions for improving the results of the first round. For example, one expert believed that safety regulation and rewards and punishments had overlapping meanings and suggested that rewards and punishments should be omitted. One expert suggested increasing the number of psychological clinical services. Another expert suggested merging the two primary indicators, organization and management. An expert also suggested increasing the social pressure indicator. In addition, experts have also revised the interpretation of individual indicators. The results of the second round of questionnaires were collected and analyzed. 
TABLE 1: Power plant worker unsafe behavior assessment indicator initial set.

\begin{tabular}{lc}
\hline Category & Indicator \\
\hline Individual personal & $\begin{array}{c}\text { Mental limit, safety awareness, personality disorder, disease, bad habit, forgetting, psychological negligence, job } \\
\text { skills, physical quality, personal interest, vision, hearing, psychological capital, physical limit, emergency } \\
\text { response, emotional management ability, attention, understanding, judgment, physical fitness, fatigue, work } \\
\text { attitude, interpersonal skills, safety knowledge, cognitive ability, tolerance, job satisfaction }\end{array}$ \\
\hline $\begin{array}{l}\text { Organizational } \\
\text { management }\end{array}$ & $\begin{array}{c}\text { Organizational atmosphere, synchronization, regulation, communication, culture, commitment, emergency } \\
\text { exercise, community relations, inspection, safety investment, readiness, leadership, rewards and punishments, } \\
\text { job design, education training, management }\end{array}$ \\
\hline Mechanical equipment & $\begin{array}{c}\text { Equipment malfunction, equipment use, equipment repairing, safety warning design, equipment reliability, } \\
\text { emergency equipment, tool distribution, protective equipment, equipment sensitivity, human-machine fitness }\end{array}$ \\
Working environment & $\begin{array}{c}\text { Visibility, high temperature, ventilation, odor, cold and wet weather, lighting, job space, monotonous, work } \\
\text { intensity, work difficulty, work time, biological hazard, noise, oxygen supply, and atmospheric pressure, } \\
\text { environmental cleanliness, environmental comfort, etc. }\end{array}$ \\
\hline Social life & Family economic status, habit, life pressure, social norm, intervention, legal issues, lifestyle \\
\hline
\end{tabular}

The third round of questionnaires was all recovered, and the expert opinions tended to be consistent. Thus, the final assessment indicator set of unsafe behavior was determined, and the results are shown in Table 2.

\subsection{Collection of Data}

2.3.1. Questionnaire Design. The data of indicators and unsafe behavior were collected by questionnaire survey. After extensive literature review, surveying of workers, and interviewing of experts, a table with 27 unsafe behavior secondary assessment indicators was obtained. The questionnaire included the following three parts.

(i) The investigation of the demographic information of the employees includes gender, age, and working hours.

(ii) In the process of unsafe behavior indicator assessment, 3 choices are set for 27 second-level indicators.

(iii) There are 6 items in the survey of unsafe behavior scores. The higher the score of each item is, the higher the risk of unsafe behavior is.

In the second and third parts, the Likert 5-level scale method was adopted. Table 3 shows the setting of questionnaire items [19-23].

2.3.2. Survey Implementation and Data Analysis. Keeping in view the geographical distribution of power plants in China and the feasibility of investigation, the scope of this study covered four power plant concentration areas in Tianjin, Yinchuan, Zoucheng, and Yangquan. Through a phone call, e-mail, and field research, the leaders of the four regional power plants were contacted. Using random sampling across different departments, online and offline questionnaires were conducted for power plant workers in these four regions. Links to online surveys were sent to contacts at the power plant, who forwarded the survey links via e-mail to the plant workers. For the offline survey, the investigators sent questionnaires to the people on the site and offered rewards for participation. The investigation began in
February 2021 and ended in June 2021. A total of 160 questionnaires were sent out and 143 were collected, and 124 were valid with an effective recovery of $86.71 \%$. The descriptive statistics of the demographic data are shown in Table 4.

As can be seen from Table 4, the age, education, homeplace, working hours, and positions of the respondents are in line with the actual situation of power plant workers, and the survey data are in line with the actual requirements. The questionnaire data were sorted out and SPSS 21.0 was used for reliability and validity analysis. The results showed that the Cronbach $\alpha$ coefficient of all dimensions of the scale was greater than 0.8 , and the Cronbach $\alpha$ coefficient of the total scale was 0.845 , indicating that the reliability of the scale met the requirements of analysis. The KMO value of the total table is 0.904 , and the KMO value of all dimensions of the scale is greater than 0.8. The results of the Bartlett spherical test are significant, indicating that the scale validity is good.

\subsection{Construction of BP Neural Network Model and Deter-} mination of the Assessment Indicator Weight. BP neural network is a multilayer feedforward neural network training based on the error backpropagation algorithm, including two learning processes: the forward propagation of signal and the backpropagation of error. BP neural network consists of the input layer, hidden layer, and output layer, and each layer is composed of several neuron nodes. The hidden layer may be one or more layers between the input and output. At first, the network propagation starts from the forward propagation. Then, the input signal acts on the hidden layer through the input layer and passes to the output layer. After a series of nonlinear changes, the actual output is obtained. When there is a big difference between the actual output and the expected output, it enters into the backpropagation process. Then, the output error is transmitted back to the input layer by the output layer acting on the hidden layer, and the weights and thresholds between the input layer and the hidden layer, as well as between the hidden layer and the output layer, are adjusted simultaneously. The training is repeated until the error is sufficiently minimal. The structure of a three-layer BP neural network is shown in Figure 2. 
TABLE 2: Unsafe behavior assessment indicator final set.

\begin{tabular}{|c|c|c|}
\hline The first-level indicator & The second-level indicator & Explanation of the indicator \\
\hline \multirow{8}{*}{ Individual personal $\mathrm{A} 1$} & $\begin{array}{l}\text { Emotional management ability } \\
\text { B1 }\end{array}$ & $\begin{array}{l}\text { The ability of employees to adjust and control emotions and relieve } \\
\text { negative emotions }\end{array}$ \\
\hline & Physical fitness B2 & Endurance, strength, acuity, and other athletic qualities of employees \\
\hline & Safety awareness B3 & $\begin{array}{c}\text { Employees' cognitive level and vigilance awareness of risks in the process of } \\
\text { work }\end{array}$ \\
\hline & Interpersonal skill B4 & $\begin{array}{c}\text { The ability of employees to interact with others and to deal with } \\
\text { interpersonal relationships }\end{array}$ \\
\hline & Job skills B5 & The employee's proficiency, experience, and technology on the job \\
\hline & Personality trait $\mathrm{B} 6$ & A behavioral tendency held by employees themselves \\
\hline & Safety knowledge B7 & $\begin{array}{c}\text { Employee's understanding and mastery of the knowledge required for safe } \\
\text { work }\end{array}$ \\
\hline & Attitude B8 & Employee's attitude towards work \\
\hline \multirow{7}{*}{$\begin{array}{l}\text { Organizational } \\
\text { management A2 }\end{array}$} & Safety supervision B9 & Supervision of unsafe behavior \\
\hline & Safety investment B10 & $\begin{array}{c}\text { The amount of time and property the enterprise needs to invest in safe } \\
\text { production }\end{array}$ \\
\hline & Organizational atmosphere B11 & A safe atmosphere between organizational groups \\
\hline & Management ability B12 & $\begin{array}{c}\text { The ability of the manager to supervise and guide the safe behavior of } \\
\text { employees }\end{array}$ \\
\hline & $\begin{array}{l}\text { The ability of communication } \\
\text { B13 }\end{array}$ & Employees' communication on safety at work \\
\hline & Educational training B14 & Cofoty educotion and troinino \\
\hline & Safety regulation B15 & Implementation of the safety management system \\
\hline \multirow{4}{*}{ Mechanical equipment A3 } & $\begin{array}{l}\text { Tool distribution B16 } \\
\text { Display design B17 }\end{array}$ & $\begin{array}{c}\text { Are tools and equipment stored safely, reasonably, and correctly } \\
\text { Display of safety signs, warning signs, etc. }\end{array}$ \\
\hline & Equipment reliability B18 & The degree of durability, safety, and reliability of the equipment \\
\hline & Labor safety devices B19 & Management of protective devices, protective facilities, safety tools, etc. \\
\hline & & \\
\hline \multirow{7}{*}{ External environment A4 } & $\begin{array}{l}\text { Physical environment } \\
\text { condition B21 }\end{array}$ & $\begin{array}{c}\text { Working site lighting, ventilation, temperature, and other physical } \\
\text { environment conditions }\end{array}$ \\
\hline & Legal issues B22 & The legal effect of the work safety law enacted by the state \\
\hline & $\begin{array}{l}\text { Psychological clinical service } \\
\text { B23 }\end{array}$ & Degree of the impact of major life events on unsafe behavior \\
\hline & Social pressure B24 & The stress of employees in social life \\
\hline & $\begin{array}{l}\text { Family economic conditions } \\
\text { B25 }\end{array}$ & mily economic status on unsafe behavior \\
\hline & Work intensity B26 & $\begin{array}{l}\text { A combination of stress, fatigue, and pain that an employee feels as a result } \\
\text { of the workload }\end{array}$ \\
\hline & Workspace B27 & The scope of activity of the employee in the process of work \\
\hline
\end{tabular}

In Figure 2, the input layer contains $m$ neurons, and the input vector $X=\left(x_{1}, x_{2}, x_{3}, \ldots, x_{j}, \ldots x_{m}\right) T$, where $x_{j}$ represents the input of the $j^{\text {th }}$ neuron in the input layer, $w_{i j}$ represents the connection weight of the $j^{\text {th }}$ neuron in the input layer and the $i^{\text {th }}$ neuron in the hidden layer, and $i=1,2$, $\ldots, n ; \theta_{i}$ shows the threshold value of the $i^{\text {th }}$ neuron in the hidden layer. The output layer contains $L$ neurons, and the output vector $d=\left(d_{1}, d_{2}, d_{3}, \ldots, d_{k}, \ldots, d_{L}\right) T, d_{k}$ represents the output of the $k^{\text {th }}$ neuron in the output layer; $w_{k i}$ represents the connection weight of the $k^{\text {th }}$ neuron in the output layer and the $i^{\text {th }}$ neuron in the hidden layer; $b_{k}$ represents the threshold value of the kth neuron in the output layer.

2.5. Determination of BP Neural Network Structure. BP neural network with one hidden layer has strong nonlinear mapping capability [17] and fast convergence speed, so this study set the number of hidden layers as 1 to establish a three-layer BP neural network model. The number of neurons in the input layer was set as 27 ; that is, 27 secondary assessment indicators (B1 B27) were used as network input. The number of neurons in the output layer is set as 1 ; that is, the sum of the 6 items of unsafe behaviors is used as the network output. The number of neurons in the hidden layer refers is computed as

$$
n=\sqrt{m+L}+q
$$

where $n$ is the number of neurons in the hidden layer, $m$ is the number of neurons in the input layer, $L$ shows the number of neurons in the output layer, and $q$ is a constant from 1 to 10 . The value range of $n$ is $6 \sim 15$.

The learning process of the BP neural network is the weight adjustment process, from which the nonlinear mapping is extracted and stored in the weight matrix, which has the characteristics of self-learning and self-adaptation [24], and is widely used in the fields of classification, recognition, approximation, regression, and prediction. The BP neural network can identify and quantify indicators and effectively map the nonlinear 
TABLE 3: Questionnaire questions and references.

\begin{tabular}{|c|c|c|c|}
\hline Indicators & $\begin{array}{c}\text { Number of secondary } \\
\text { indicators }\end{array}$ & $\begin{array}{c}\text { Item } \\
\text { number }\end{array}$ & Item example \\
\hline Individual personal $\mathrm{A} 1$ & 8 & 24 & $\begin{array}{l}\text { (1) When confronted with unexpected problems, I can deal with } \\
\text { them calmly } \\
\text { (2) I know the safety knowledge of my position very well } \\
\text { (3) I am very conscientious and responsible during my work }\end{array}$ \\
\hline $\begin{array}{l}\text { Organizational management } \\
\text { A2 }\end{array}$ & 7 & 21 & $\begin{array}{l}\text { (1) I enjoy discussing job skills with my colleagues } \\
\text { (2) The work unit constrains us strictly in accordance with the } \\
\text { safety system } \\
\text { (3) Work units often organize staff to carry out safety education } \\
\text { activities }\end{array}$ \\
\hline Mechanical equipment A3 & 5 & 15 & $\begin{array}{l}\text { (1) The work unit can update and maintain the equipment in } \\
\text { time } \\
\text { (2) When I'm done with my tools, I put them in a proper place } \\
\text { (3) I can use tools and equipment correctly }\end{array}$ \\
\hline External environment A4 & 7 & 21 & $\begin{array}{l}\text { (1) I can operate safely in poor working conditions } \\
\text { (2) Family matters tend to cause me to break the rules } \\
\text { (3) The workload is so heavy that I may not follow the rules }\end{array}$ \\
\hline Unsafe behavior & - & 6 & $\begin{array}{l}\text { (1) I may engage in unsafe behavior to save time and effort } \\
\text { (2) I may unconsciously stand or sit in dangerous areas } \\
\text { (3) I might use my hands instead of machines }\end{array}$ \\
\hline
\end{tabular}

TABle 4: Descriptive statistics of the demographic data.

\begin{tabular}{|c|c|c|c|}
\hline Variable & Category & Number of people & Proportion (\%) \\
\hline \multirow{2}{*}{ Gender } & Male & 96 & 77.42 \\
\hline & Female & 28 & 22.58 \\
\hline \multirow{3}{*}{ Age } & Age 25 and below & 35 & 28.23 \\
\hline & $26-35$ years old & 53 & 42.74 \\
\hline & Age 36 and above & 36 & 29.03 \\
\hline \multirow{3}{*}{ Education } & Senior high school and below & 33 & 26.61 \\
\hline & Junior college and undergraduate & 50 & 40.32 \\
\hline & Postgraduate or above & 41 & 33.07 \\
\hline \multirow{3}{*}{ Homeplace } & City & 28 & 22.58 \\
\hline & Villages and towns & 49 & 39.52 \\
\hline & Country & 47 & 37.90 \\
\hline \multirow{4}{*}{ Working hours } & 3 years and below & 27 & 21.77 \\
\hline & $4-5$ years & 32 & 25.81 \\
\hline & $6-10$ years & 21 & 16.94 \\
\hline & 11 years and above & 44 & 35.48 \\
\hline \multirow{7}{*}{ Operating post } & Boiler & 26 & 20.97 \\
\hline & Motor machine & 23 & 18.55 \\
\hline & Electrical engineering & 20 & 16.13 \\
\hline & Material & 14 & 11.29 \\
\hline & Environmental chemistry & 16 & 12.90 \\
\hline & New energy resources & 19 & 15.32 \\
\hline & Else & 6 & 4.84 \\
\hline
\end{tabular}

relationship among assessment indicators and the internal relationship between each indicator and the assessment object. Moreover, it is a kind of cause-and-effect method. After training and learning known samples, an adaptive mechanism of network weights is established, and the trained network weights are used to calculate the indicator weights, making the weights more efficient. In addition, it can also effectively reduce the influence of human subjective factors, making the weight result more effective. To determine the weights of indicators, the following steps were employed using BP neural network. (i) The input (assessment indicator data) and output (assessment object) of the network were determined with sample data.

(ii) The BP neural network model is established and network structure is determined.

(iii) The network is trained and the weight of the network connection and threshold is adjusted.

(iv) The performance of the network is tested.

(v) After the network performance is improved, the indicator weight is calculated according to the 


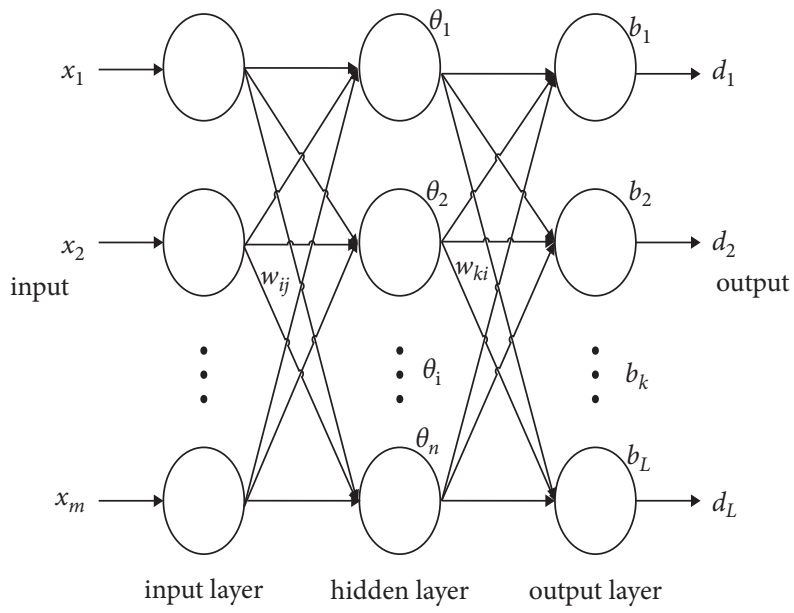

FIgURE 2: Three-layer BP neural network structure.

connection weight of the input layer and the hidden layer and is computed as

$$
v_{j}=\frac{\sum_{i=1}^{n}\left|w_{i j}\right|}{\sum_{l=1}^{m} \sum_{i=1}^{n}\left|w_{i l}\right|}, \quad j=1,2, \ldots, m,
$$

where $v_{j}$ is the weight of the $j^{\text {th }}$ neuron in the input layer; $m$ is the number of neurons in the input layer, $n$ shows the number of neurons in the hidden layer, $w_{i j}$ is the connection weight between the $j^{\text {th }}$ neuron in the input layer and the $i^{\text {th }}$ neuron in the hidden layer, and $w_{i l}$ indicates the connection weight of the $l^{\text {th }}$ neuron in the input layer and the $i^{\text {th }}$ neuron in the hidden layer.

\section{Results}

\subsection{BP Neural Network Simulation}

3.1.1. Data Normalization. Before training the BP neural network, the 124 data samples were normalized to eliminate outliers and accelerate the convergence speed of the network. The goal of normalization is to convert the values of numeric data columns in the dataset to a common standard scale, without altering variations in the ranges of values $[13,14]$. For machine learning, every dataset does not require normalization. It is required only when features have different ranges. There are different types of data normalization. In this study, the min-max method of data normalization is employed to obtain a standard set of values. Using data normalization all the data samples were normalized into the range of 0 and 1 . The data normalization results are shown in Table 5 .

3.1.2. Network Training and Testing. MatlabR2015 software was used to train and test the BP neural network. After repeated calculation of the normalized data samples, when the number of neurons in the hidden layer was 13, the network error and the number of iterative steps were small; therefore, the number of neurons in the hidden layer was set as 13, and the BP neural network model with "27-13-1" structure was developed. All the 124 normalized data samples were divided into two parts comprising a training set (110 data samples) and a testing set (14 data samples). After repeated tests, the network parameters were set as shown in Table 6. When the training times are 169, the mean square error (MSE) meets the accuracy requirements, as shown in Figure 3.

After the training, the test set was input into the trained network for simulation, and the comparison results between the real number and predicted number of the test set were obtained, as shown in Figure 4.

As can be seen from Figure 4, the difference between the real number and the predicted number in the test set is sufficiently small, indicating that the trained BP neural network can accurately reflect the complex relationship between assessment indicators and unsafe behaviors.

3.2. Determination of Weights of Assessment Indicators. Backpropagation is the heart of neural network training. It is the method of fine-tuning the weights of a neural network based on the error rate obtained in the previous epoch (i.e., iteration). Proper tuning of the weights allows you to reduce error rates and make the model reliable by increasing its generalization. It can be seen from the previous section that, through the self-learning function of the BP neural network, the connection weights and thresholds between each network layer have met the accuracy requirements. MATLAB software was used to obtain the connection weight between the input layer and the hidden layer of the trained BP neural network, as shown in Table 7.

Using equation (2), the weights of the 27 secondary assessment indicators of the plant workers' unsafe behaviors are obtained by calculating the data in Table 7 . The results are shown in Table 8 .

As can be seen from Table 8 the weights of all indicators are greater than zero, indicating that all the 27 second-level indicators have an impact on unsafe behaviors. Therefore according to the weights we get $\mathrm{B} 26>\mathrm{B} 3>\mathrm{B} 19>\mathrm{B} 2>\mathrm{B} 20>$ $\mathrm{B} 12>\mathrm{B} 9>\mathrm{B} 24>\mathrm{B} 7>\mathrm{B} 18>\mathrm{B} 11>\mathrm{B} 1>\mathrm{B} 16>\mathrm{B} 27>\mathrm{B} 21>$ 
TABLE 5: Normalized processing results of questionnaire data.

\begin{tabular}{|c|c|c|c|c|c|c|c|c|c|c|c|c|c|}
\hline \multirow{2}{*}{ Assessment indicator } & \multicolumn{13}{|c|}{ Normalized sample data } \\
\hline & 1 & 2 & 3 & 4 & 5 & 6 & 7 & 8 & 9 & 10 & $\ldots \ldots$ & 123 & 124 \\
\hline $\mathrm{B} 1$ & 0.8 & 0.7 & 0.5 & 0.5 & 0.7 & 0.7 & 1.0 & 0.5 & 0.6 & 0.6 & $\ldots \ldots$ & 0.8 & 0.5 \\
\hline B2 & 0.5 & 0.5 & 0.9 & 0.7 & 0.5 & 0.5 & 0.8 & 0.6 & 0.6 & 0.7 & $\ldots \ldots$ & 0.6 & 0.7 \\
\hline B3 & 0.8 & 0.8 & 0.6 & 0.8 & 0.6 & 0.5 & 0.6 & 0.5 & 0.5 & 0.6 & $\ldots \ldots$ & 0.6 & 0.5 \\
\hline B4 & 0.7 & 0.5 & 0.5 & 0.6 & 0.5 & 0.4 & 0.7 & 0.3 & 0.6 & 0.6 & $\ldots \ldots$ & 0.6 & 0.5 \\
\hline B5 & 0.6 & 0.8 & 0.7 & 0.9 & 0.7 & 0.8 & 0.6 & 0.6 & 0.4 & 0.6 & $\ldots \ldots$ & 0.5 & 0.6 \\
\hline B6 & 0.7 & 0.8 & 0.6 & 0.9 & 0.6 & 0.5 & 0.5 & 0.5 & 0.5 & 0.7 & $\ldots \ldots$ & 0.7 & 0.7 \\
\hline B7 & 0.5 & 0.4 & 0.6 & 0.5 & 0.5 & 0.7 & 0.9 & 0.7 & 0.6 & 0.5 & $\ldots \ldots$ & 0.7 & 0.4 \\
\hline B8 & 0.5 & 0.5 & 0.6 & 0.8 & 0.5 & 0.6 & 0.6 & 0.4 & 0.4 & 0.6 & $\ldots \ldots$ & 0.5 & 0.6 \\
\hline B9 & 0.5 & 0.5 & 0.5 & 0.5 & 0.4 & 0.7 & 0.7 & 0.5 & 0.5 & 0.6 & $\ldots \ldots$ & 0.7 & 0.4 \\
\hline B10 & 0.5 & 0.5 & 0.6 & 0.6 & 0.7 & 0.5 & 0.8 & 0.6 & 0.6 & 0.5 & $\ldots \ldots$ & 0.7 & 0.6 \\
\hline B11 & 0.7 & 0.8 & 0.6 & 0.6 & 0.5 & 0.6 & 0.8 & 0.5 & 0.4 & 0.7 & $\ldots \ldots$ & 0.5 & 0.6 \\
\hline B12 & 0.8 & 0.7 & 0.7 & 0.5 & 0.5 & 0.8 & 0.6 & 0.7 & 0.6 & 0.7 & $\ldots \ldots$ & 0.7 & 0.5 \\
\hline B13 & 0.6 & 0.4 & 0.5 & 0.6 & 0.7 & 0.4 & 0.6 & 0.4 & 0.5 & 0.7 & $\ldots \ldots$ & 0.6 & 0.6 \\
\hline B14 & 0.8 & 0.6 & 0.8 & 0.7 & 0.7 & 0.8 & 0.7 & 0.7 & 0.6 & 0.8 & $\ldots \ldots$ & 0.8 & 0.7 \\
\hline B15 & 0.7 & 0.8 & 0.7 & 0.6 & 0.7 & 0.6 & 0.7 & 0.6 & 0.6 & 0.8 & $\ldots \ldots$ & 0.8 & 0.6 \\
\hline B16 & 0.5 & 0.5 & 0.6 & 0.8 & 0.5 & 0.5 & 0.4 & 0.5 & 0.4 & 0.7 & $\ldots \ldots$ & 0.7 & 0.4 \\
\hline B17 & 0.6 & 0.6 & 0.4 & 0.4 & 0.5 & 0.4 & 0.6 & 0.4 & 0.4 & 0.5 & $\ldots \ldots$ & 0.4 & 0.6 \\
\hline B18 & 0.4 & 0.5 & 0.7 & 0.5 & 0.4 & 0.4 & 0.5 & 0.5 & 0.4 & 0.6 & $\ldots \ldots$ & 0.7 & 0.5 \\
\hline B19 & 0.6 & 0.5 & 0.5 & 0.5 & 0.7 & 0.5 & 0.5 & 0.5 & 0.5 & 0.6 & $\ldots \ldots$ & 0.7 & 0.8 \\
\hline B20 & 0.5 & 0.5 & 0.5 & 0.6 & 0.4 & 0.4 & 0.6 & 0.5 & 0.6 & 0.4 & $\ldots \ldots$ & 0.4 & 0.4 \\
\hline B21 & 0.7 & 0.6 & 0.5 & 0.5 & 0.6 & 0.6 & 0.4 & 0.4 & 0.5 & 0.5 & $\ldots \ldots$ & 0.4 & 0.7 \\
\hline B22 & 0.7 & 0.6 & 0.7 & 0.8 & 0.7 & 0.6 & 0.7 & 0.9 & 0.6 & 0.8 & $\ldots \ldots$ & 0.7 & 0.8 \\
\hline B23 & 0.4 & 0.4 & 0.5 & 0.4 & 0.7 & 0.6 & 0.4 & 0.4 & 0.4 & 0.3 & $\ldots \ldots$ & 1.0 & 0.7 \\
\hline B24 & 0.5 & 0.6 & 0.5 & 0.4 & 0.4 & 0.8 & 0.8 & 0.3 & 0.6 & 0.3 & $\ldots \ldots$ & 0.6 & 0.8 \\
\hline B25 & 0.3 & 0.7 & 0.7 & 0.4 & 0.2 & 0.5 & 0.5 & 0.2 & 0.5 & 0.4 & $\ldots \ldots$ & 0.5 & 0.7 \\
\hline B26 & 0.7 & 0.5 & 0.6 & 0.6 & 0.6 & 0.4 & 0.4 & 0.7 & 0.7 & 0.4 & $\ldots \ldots$ & 0.6 & 0.5 \\
\hline $\mathrm{B} 27$ & 0.6 & 0.7 & 0.7 & 0.4 & 0.5 & 0.7 & 0.7 & 0.6 & 0.4 & 0.5 & $\ldots \ldots$ & 0.4 & 0.5 \\
\hline Unsafe behavior & 0.4 & 0.3 & 0.3 & 0.4 & 0.5 & 0.4 & 0.3 & 0.5 & 0.6 & 0.5 & $\ldots \ldots$ & 0.3 & 0.4 \\
\hline
\end{tabular}

TABLE 6: Network parameter settings.

\begin{tabular}{lc}
\hline Function name & $\begin{array}{c}\text { Function type/parameter } \\
\text { value }\end{array}$ \\
\hline Training function & trainlm \\
Hidden layer activation function & tansig \\
Output layer activation function & purelin \\
Performance function & mse \\
Maximum number of training steps & epochs $=5000$ \\
Learning rate & 0.05 \\
Target error & 0.001 \\
\hline
\end{tabular}

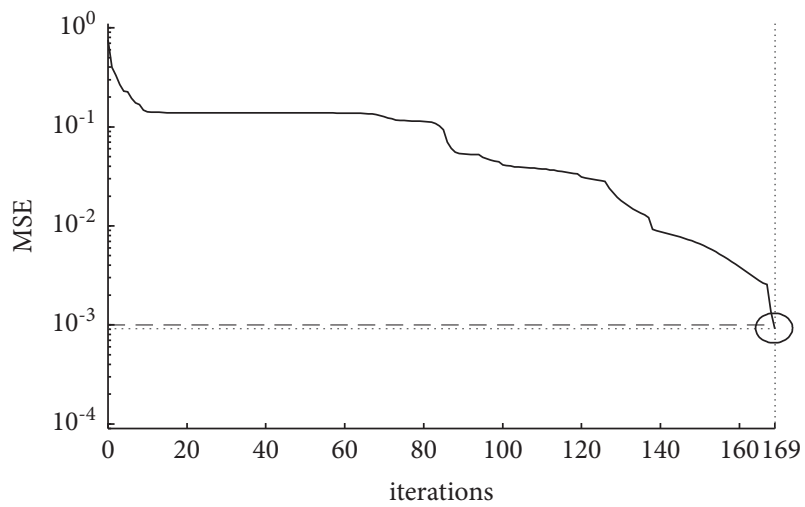

FIGURE 3: Network error change.
$\mathrm{B} 13>\mathrm{B} 25>\mathrm{B} 8>\mathrm{B} 6>\mathrm{B} 15>\mathrm{B} 14>\mathrm{B} 23>\mathrm{B} 5>\mathrm{B} 22>\mathrm{B} 4>$ $\mathrm{B} 17>\mathrm{B} 10$. Therefore, for workers in power plants, the prevention and control of their unsafe behaviors should start from the importance indicator and take corresponding prevention and control measures, such as reducing workers' work intensity (B26), enhancing workers' safety awareness (B3), and strengthening the management of labor protection devices (B19).

\section{Discussion}

Electrical accidents can cause serious injuries in the workplace. However, the control measures that prevent these hazards require careful management, attention to detail, and technical competence. According to the Accident Causation theory, the unsafe behavior of people and the unsafe state of objects in the electric workplace are the two major factors leading to the occurrence of electric power accidents [25]. Since the unsafe state of objects is mostly due to human factors, the unsafe behavior of people is the main cause of accidents [26]. For on-site workers in power plants, they face not only common accidents such as object hit, falling from a height, and fire, but also electrical hazards such as electric shock, static electricity, radiofrequency radiation, and circuit faults. Compared with other industries, there are more dangers of accidents in electric power plants, and the danger of unsafe behaviors is also greater [27]. When the on-site workers in power plants are in a complex site environment 


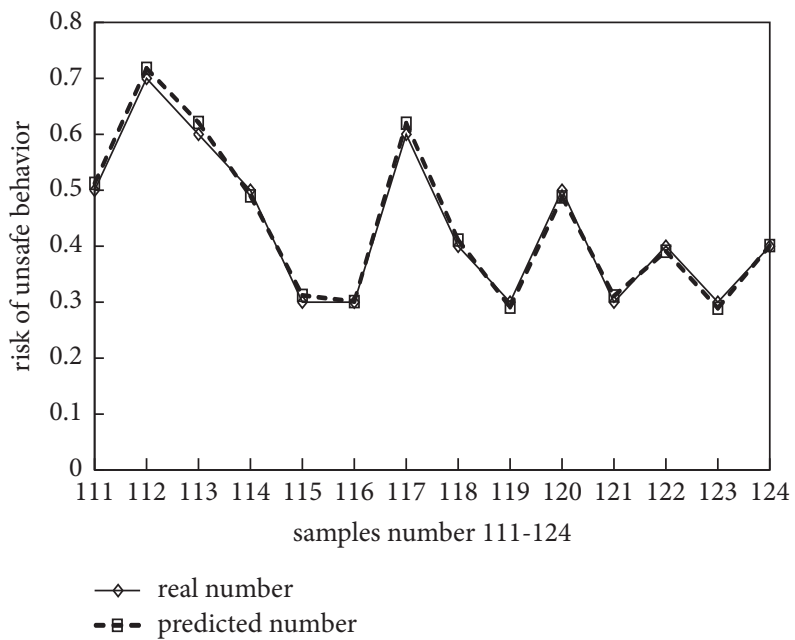

FIGURE 4: Comparison results of a real number and predicted number.

TABLE 7: Connection weights of the input layer and hidden layer.

\begin{tabular}{|c|c|c|c|c|c|c|c|c|c|c|c|c|c|}
\hline \multirow{2}{*}{ Input layer } & \multicolumn{13}{|c|}{ Hidden layer } \\
\hline & 1 & 2 & 3 & 4 & 5 & 6 & 7 & 8 & 9 & 10 & 11 & 12 & 13 \\
\hline 1 & -0.2952 & 0.0585 & 0.3663 & .9917 & -0.4327 & 0.3371 & 0.5950 & 0.2663 & -0.0780 & -0.1982 & 0.2817 & -0.2588 & -0.0395 \\
\hline 2 & -0.2869 & 0.0892 & 0.2203 & 0.5842 & & 0.2336 & & & & 3072 & & & \\
\hline 3 & 0.7548 & 0.5250 & 0.2235 & -0.6407 & 0.4147 & -0.8581 & & -0.4803 & -0.1793 & -0.3932 & 0.4267 & -0.2042 & 0.3136 \\
\hline 4 & 0.0073 & -0.0717 & -0.2040 & 0.4151 & 0.0068 & -0.6182 & 0.2875 & -0.1398 & 0.2135 & -0.1455 & 0.7740 & 0.0560 & 0.2669 \\
\hline 5 & -0.0388 & -0.0083 & -1.1526 & 0.0992 & -0.0135 & -0.0156 & -0.5322 & -0.0431 & -0.4243 & -0.5547 & 0.2701 & 0.1408 & -0.1827 \\
\hline 6 & 0.4757 & -0.0857 & 0.5354 & 0.0996 & 0.4099 & -0.6229 & 0.1425 & -0.1 & 0.0 & 0.1 & -0.3 & -0.2 & -0.3757 \\
\hline 7 & 1050 & 0.4414 & -0.1166 & 0.1225 & 4 & -0.8 & & -0. & 0.7 & -0 . & & & \\
\hline 8 & 0.5886 & -0.0302 & 0.1164 & -0.0657 & -1. & -0.1 & 0.4 & 0.3 & -0.2098 & -0.1 & 0.2397 & -0 . & 0.2027 \\
\hline 9 & -0.2902 & 0.4937 & -0.3174 & 0.3021 & -0.5782 & -0.4748 & -0.1900 & 0.4145 & 0.5013 & 0.0325 & & 0.4790 & 0.3461 \\
\hline 10 & 0.1632 & 0.00 & 0.2526 & -0.2049 & -0.1413 & -0.5245 & -0.1 & 0.3 & 0.1 & 0.0 & 50 & 0.1 & -0.3082 \\
\hline 11 & 0.2115 & 0.45 & & -0.27 & -0.3 & 0.2991 & & 0.1 & & -0 & 50 & -0 & 19 \\
\hline 12 & -0.3240 & 0.0945 & & & & & & -0 . & & -0 . & -0 & -0 . & -1.0 \\
\hline 13 & -0.0243 & -0.2433 & 10 & 4 & & 42 & & & -0. & -0 . & & -0 . & -0.2132 \\
\hline 14 & & 0.2992 & -0.6588 & 0.2533 & 0.0358 & -0.1807 & 0.3 & -0 . & -0.1 & -0.0 & & & 0.2438 \\
\hline 15 & -0.4343 & -0.0 & & & & & & & 28 & -0 & -0 & -0 & -0 \\
\hline 16 & -0.1226 & 030 & -0.0 & 9 & -0.3 & 1 & -0 & 77 & 0.2 & & -0 . & & 0.8375 \\
\hline 17 & & 0.3043 & -0.0286 & -0.0189 & 0.2454 & -0.3051 & -0.3599 & 0.2706 & -0.2475 & & & -0 & -0.0610 \\
\hline 18 & & & & & -0.3805 & 0.3118 & -0.5222 & -0.1878 & 0.0568 & & 0.3501 & -0.5 & -0.0819 \\
\hline 19 & -0.3 & -0.6 & & & & & & & & & -0 & & -0 \\
\hline 20 & 0.4921 & 0.1508 & -0.1789 & 0.7271 & -0.4049 & -0.1384 & 44 & 0.0878 & 26 & 56 & 0.2186 & -0.2743 & -0.3253 \\
\hline 21 & -0.5855 & -0.1424 & 0.0569 & -0.0642 & 0.8577 & -0.1279 & 0.0514 & 0.4017 & 0.5526 & -0.0053 & -0.3108 & -0.5606 & 0.2282 \\
\hline 22 & 0.2434 & 0.7988 & -0.0103 & -0.3708 & -0.0187 & -0.6294 & 0.1310 & -0.3730 & -0.1851 & 0.1337 & -0.0605 & -0.2389 & -0.2441 \\
\hline 23 & -0.5077 & -0.0 & -0.0773 & & -0.6 & & & & & & 0.2662 & -0.1403 & -0.0962 \\
\hline 24 & 0.1316 & -0.0407 & -0.0789 & 0.2234 & 0.3939 & 0.0673 & -0.6658 & 0.5578 & -0.3500 & -0.6449 & -0.8370 & -0.3285 & -0.3830 \\
\hline 25 & -0.1138 & -0.3791 & 0.4841 & 0.1840 & 0.0061 & -0.3553 & 0.5431 & 0.1653 & 0.5348 & 0.2657 & 0.1775 & -0.2424 & -0.4098 \\
\hline 26 & -0.1263 & -0.7877 & -0.6744 & 0.0036 & 0.3495 & 0.1756 & 0.4227 & -0.2467 & 0.1085 & -0.4655 & 1.1550 & -0.6489 & 0.6749 \\
\hline 27 & -0.5675 & 0.0194 & -0.2914 & -0.3810 & -0.0378 & -0.7788 & 0.3263 & -0.5978 & 0.3844 & -0.0161 & 0.0934 & 0.4167 & 0.0683 \\
\hline
\end{tabular}

for a long time, accompanied by heavy physical and mental work, it will lead to the unsafe state of workers easily falling into negative emotions [28], physical fatigue [29], and consciousness decline [30], resulting in increased potential safety accidents. Nowadays, with the development of safety technology, the safety performance of power plants is improving. However, due to the characteristics and limits of human beings in physiology, psychology, and spirit, the unsafe behavior of human beings is difficult to control and unstable. Therefore, to ensure the safe operation of power plant workers, it is necessary to comprehensively evaluate their unsafe behaviors.

There were few researches on the analysis of unsafe behavior of power plant workers and few researches on the unsafe behavior from the perspective of assessment indicator [31]. Studies on unsafe behaviors from the perspective of assessment indicators are rare. Establishing a scientific and comprehensive assessment indicator system of workers' unsafe behavior in power plants is the basis of monitoring and evaluating workers' unsafe state and an important way 
TABLE 8: Calculation results of the weight of the assessment indicator system.

\begin{tabular}{|c|c|c|c|c|c|c|c|c|c|}
\hline \multicolumn{10}{|l|}{ Indicator } \\
\hline & B1 & B2 & B3 & B4 & B5 & B6 & B7 & B8 & B9 \\
\hline Weight & 0.0374 & 0.0446 & 0.0487 & 0.0286 & 0.0310 & 0.0332 & 0.0396 & 0.0339 & 0.0421 \\
\hline Ranking & 12 & 4 & 2 & 25 & 23 & 19 & 9 & 18 & 7 \\
\hline & B10 & B11 & B12 & B13 & B14 & B15 & B16 & B17 & B18 \\
\hline Weight & 0.0264 & 0.0376 & 0.0425 & 0.0349 & 0.0320 & 0.0321 & 0.0360 & 0.0280 & 0.0386 \\
\hline Ranking & 27 & 11 & 6 & 16 & 21 & 20 & 13 & 26 & 10 \\
\hline & B19 & B20 & B21 & B22 & B23 & B24 & B25 & B26 & B27 \\
\hline Weight & 0.0486 & 0.0428 & 0.0352 & 0.0306 & 0.0315 & 0.0419 & 0.0344 & 0.0520 & 0.0355 \\
\hline Ranking & 3 & 5 & 15 & 24 & 22 & 8 & 17 & 1 & 14 \\
\hline
\end{tabular}

to effectively prevent workers' unsafe behavior. This study proposed an assessment method for power plant workers unsafe behavior based on BP neural network. An assessment indicator system was composed of 4 first-level indicators and 27 second-level indicators. The assessment indicators were taken as network input and unsafe behaviors as network output. Finally, the weights of 27 secondary indicators are obtained through training of the model. And a three-layer BP neural network assessment model of "27-13-1" is developed.

BP neural network is highly applicable in the field of assessment and prediction [12-16]. To overcome the shortcomings of traditional assessment methods, such as difficulty in defining the relationship between indicators, strong subjectivity in weight determination, and difficulty in reproducing the assessment results [32], this study used the neural networks algorithm to determine the indicator weight. Besides, it can carry on numerous trainings for huge amounts of data. Therefore, the assessment weight is fit, and the assessment model is more effective. In this study, the power plant workers in Tianjin, Yinchuan, Zoucheng, and Yangquan cities of China were taken as examples. The corresponding indicator data were collected through questionnaires and the corresponding indicator weights were calculated using a neural network. Based on the indicator weight, managers can propose specific unsafe behavior prevention and control measures from four aspects: individual personal, organizational management, mechanical equipment, and external environment. For example, at the individual level, it was found that $\mathrm{B} 3>\mathrm{B} 2>\mathrm{B} 7>\mathrm{B} 1>\mathrm{B} 8>\mathrm{B} 6>\mathrm{B} 5>\mathrm{B} 4$. Therefore, managers can emphasize strategies aimed at enhancing workers' safety awareness, physical fitness, safety knowledge, and emotional management ability. In addition, it can be seen from the comparison in Figure 4 that the error between the predicted number and the real number in the test set is sufficiently small, indicating that the trained BP neural network can accurately reflect the complex relationship between assessment indicators and unsafe behaviors.

The occurrence of unsafe behaviors of power plant workers is inherently complicated [4]. It is greatly affected by individual, organization management, mechanical equipment, and external environment. By constructing the assessment indicator system, the influencing factors of unsafe behaviors are selected and the assessment basis is given.
Meanwhile, the differences of unsafe behaviors under different indicators can be preliminarily determined. BP neural network model is used to explore the nonlinear complex relationship between different indicators and unsafe behavior, which is of great significance to the prevention of unsafe behavior. Safety managers can choose different indicators according to their own needs and judge whether there is regularity between them through the weight calculation results, to improve the efficiency of safety management and promote the promotion of experiential safety management to preventive safety management.

\section{Conclusion}

This study established a comprehensive and accurate assessment indicator system for the unsafe behavior of power plant workers, which comprised individual, organization and management, mechanical equipment, external environment, 4 first-level indicators, and 27 secondary indicators. Then a backpropagation BP neural network model of unsafe behavior assessment was established, and the model was simulated and analyzed. The weight of the assessment indicators was determined. A three-layer feedforward backpropagation neural network assessment model of " 27 13-1" was found to be a suitable model. After training and learning, BP neural network method can demonstrate the nonlinear complex relationship between the assessment indicator and the unsafe behavior of power plant workers. The BP neural network method is used to determine the weight of unsafe behavior assessment indicators, and according to the weight value, prevention and control measures can be taken to reduce the occurrence of unsafe behavior. This work places the foundation for future model development, to further satisfy the demands from different departments within the power plants by adding or deleting the number of factors. There are some limitations associated with this study. First, 27 indicators were not sufficient to fully understand the root cause of power plants accidents; more indicators would be investigated. Second, because the real world is complex and dynamic, the future model should be adjustable and capable of treating data automatically. Third, the task of the control room is extremely important; therefore, the unsafe behaviors of control room engineers should be further studied. 


\section{Data Availability}

The data used to support the findings of this study are included within the article.

\section{Conflicts of Interest}

The authors declare that they have no conflicts of interest.

\section{References}

[1] G. Fu, L. Zhou, J. Wang, and M. Shi, "Analysis of an explosion accident at Dangyang power plant in Hubei, China: causes and lessons learned," Safety Science, vol. 102, pp. 134-143, 2018.

[2] X. Meng, Q. Liu, X. Luo, and X. Zhou, "Risk assessment of the unsafe behaviours of humans in fatal gas explosion accidents in China's underground coal mines," Journal of Cleaner Production, vol. 210, pp. 970-976, 2019.

[3] J. Shi, D. Y. Chang, P. Zheng, G. L. Li, and J. Y. Zhou, "Study on the propagation of unsafe behavior of laboratory personnel based on SD-SEIR models," Complex Systems and Complexity Science, vol. 18, no. 3, pp. 67-74, 2021.

[4] R. Tong, Y. Zhang, P. Cui, C. Zhai, M. Shi, and S. Xu, "Characteristic analysis of unsafe behavior by coal miners: multi-dimensional description of the pan-scene data," International Journal of Environmental Research and Public Health, vol. 15, no. 8, p. 1608, 2018.

[5] M. Malakoutikhah, M. Jahangiri, M. Alimohammadlou, S. A. Faghihi, and M. Kamalinia, "The factors affecting unsafe behaviors of Iranian workers: a qualitative study based on grounded theory," Safety and Health at Work, vol. 12, no. 3, pp. 339-345, 2021.

[6] H. Mokarami, S. S. Alizadeh, T. Rahimi Pordanjani, and S. Varmazyar, "The relationship between organizational safety culture and unsafe behaviors, and accidents among public transport bus drivers using structural equation modeling," Transportation Research Part F: Traffic Psychology and Behaviour, vol. 65, pp. 46-55, 2019.

[7] M. Shin, H.-S. Lee, M. Park, M. Moon, and S. Han, “A system dynamics approach for modeling construction workers' safety attitudes and behaviors," Accident Analysis \& Prevention, vol. 68, pp. 95-105, 2014.

[8] M.-k. Li, Y.-k. Liu, M.-j. Peng, C.-l. Xie, and L.-q. Yang, "The digital simulation and fuzzy evaluation to reduce the likelihood of unsafe behavior in nuclear decommissioning," Annals of Nuclear Energy, vol. 119, pp. 331-341, 2018.

[9] R. P. Tong, H. W. Li, B. L. Zhang, X. Y. Yang, and X. F. Ma, "Modeling of unsafe behavior risk assessment: a case study of Chinese furniture manufacturers," Safety Science, vol. 136, Article ID 105157, 2021.

[10] T. R. Zhang, D. M. Shen, S. W. Zheng, Z. P. Liu, X. D. Qu, and D. Tao, "Predicting unsafe behaviors at nuclear power plants: an integration of theory of planned behavior and technology acceptance model," International Journal of Industrial Ergonomics, vol. 80, Article ID 103047, 2020.

[11] K. Yu, Q. Cao, C. Xie, N. Qu, and L. Zhou, "Analysis of intervention strategies for coal miners' unsafe behaviors based on analytic network process and system dynamics," Safety Science, vol. 118, pp. 145-157, 2019.

[12] M. C. Li and J. C. Wang, "Intelligent recognition of safety risk in metro engineering construction based on BP neural network," Mathematical Problems in Engineering, vol. 2021, Article ID 5587027, 10 pages, 2021.
[13] T.-Y. Tseng and Q. Luo, "Company employee quality evaluation model based on BP neural network," Journal of Intelligent and Fuzzy Systems, vol. 40, no. 4, pp. 5883-5892, 2021.

[14] J.-X. Han, M.-Y. Ma, and K. Wang, "Product modeling design based on genetic algorithm and BP neural network," Neural Computing \& Applications, vol. 33, no. 9, pp. 4111-4117, 2021.

[15] J. Y. Feng, B. Y. Yuan, X. Li, D. Tian, and W. S. Mu, "Evaluation on risks of sustainable supply chain based on optimized BP neural networks in the fresh grape industry," Computers and Electronics in Agriculture, vol. 183, Article ID 105988, 2021

[16] L. M. Zhang, X. X. Zhang, Z. M. Li, and H. Lu, “Assessment of think tank's information dissemination capacity on WeChat public platform based on BP neural network," Information Studies: Theory \& Application, vol. 41, no. 10, pp. 93-99, 2018.

[17] W. Wang, R. Tang, C. Li, P. Liu, and L. Luo, "A BP neural network model optimized by mind evolutionary algorithm for predicting the ocean wave heights," Ocean Engineering, vol. 162, pp. 98-107, 2018.

[18] J. Pei, W. Liu, and L. Han, "Research on evaluation index system of Chinese city safety resilience based on Delphi method and cloud model," International Journal of Environmental Research and Public Health, vol. 16, no. 20, pp. 1-16, 2019.

[19] J. Zhang and $\mathrm{C}$. $\mathrm{Wu}$, "The influence of dispositional mindfulness on safety behaviors: a dual process perspective," Accident Analysis \& Prevention, vol. 70, pp. 24-32, 2014.

[20] C. K. H. Hon, A. P. C. Chan, and M. C. H. Yam, "Relationships between safety climate and safety performance of building repair, maintenance, minor alteration, and addition (RMAA) works," Safety Science, vol. 65, pp. 10-19, 2014.

[21] E. J. Lee, B. C. Ko, and J.-Y. Nam, "Recognizing pedestrian's unsafe behaviors in far-infrared imagery at night," Infrared Physics \& Technology, vol. 76, pp. 261-270, 2016.

[22] G. Ghic, C. G. Bentoiu, and A. Moraru, "Developing questionnaires and interview techniques on the standard of living and quality of life-teaching and practical approaches," Procedia-Social and Behavioral Sciences, vol. 116, pp. 5140-5142, 2014.

[23] I. Mohammadfam, F. Ghasemi, O. Kalatpour, and A. Moghimbeigi, "Constructing a Bayesian network model for improving safety behavior of employees at workplaces," Applied Ergonomics, vol. 58, pp. 35-47, 2017.

[24] W. Dai and P. Hu, "Application of BP neural network in the analytic hierarchy process of person-post evaluation model," The Journal of Supercomputing, vol. 76, no. 2, pp. 897-914, 2020.

[25] I. Park, J. Kim, S. Han, and C. Hyun, "Analysis of fatal accidents and their causes in the Korean construction industry," Sustainability, vol. 12, no. 8, 2020.

[26] C. Zhou, R. Chen, S. N. Jiang et al., "Human dynamics in nearmiss accidents resulting from unsafe behavior of construction workers," Physica A: Statistical Mechanics and Its Applications, vol. 530, Article ID 121495, 2019.

[27] D. Tao, Z. Liu, X. Diao, H. Tan, X. Qu, and T. Zhang, "Antecedents of self-reported safety behaviors among commissioning workers in nuclear power plants: the roles of demographics, personality traits and safety attitudes," Nuclear Engineering and Technology, vol. 53, no. 5, pp. 1454-1463, 2021.

[28] C. Min, F. Shen, W. Yu, and Y. Chu, "The relationship between government trust and preventive behaviors during the COVID-19 pandemic in China: exploring the roles of 
knowledge and negative emotion," Preventive Medicine, vol. 141, Article ID 106288, 2020.

[29] D. R. Chebat, L. Lemarié, B. Rotnemer, T. Talbi, and M. Wagner, "The young and the reckless: social and physical warning messages reduce dangerous driving behavior in a simulator," Journal of Retailing and Consumer Services, vol. 63, Article ID 102701, 2021.

[30] G. Ye, H. Z. Yue, J. J. Yang et al., "Understanding the sociocognitive process of construction workers' unsafe behaviors: an agent-based modeling approach," International Journal of Environmental Research and Public Health, vol. 17, no. 5, 2020.

[31] X. Yu, D. Zhan, L. Liu, H. Lv, L. Xu, and J. Du, "A privacy-preserving cross-domain healthcare wearables recommendation algorithm based on domain-dependent and domain-independent feature fusion," IEEE Journal of Biomedical and Health Informatics, p. 1, 2021.

[32] M. Yu, T. Quan, Q. Peng, X. Yu, and L. Liu, "A model-based collaborate filtering algorithm based on stacked AutoEncoder," Neural Computing \& Applications, 2021. 